Nota Técnica de la

Agencia Estatal de Meteorología N ${ }^{0} 11$

\title{
Análisis de la radiación solar ultravioleta en \\ Tenerife como posible herramienta para el \\ uso de Tratamientos Fotocatalíticos de Oxidación Avanzada en la depuración de aguas residuales
}

$M^{\mathrm{a}}$ Isabel García Álvarez

Centro de Investigación Atmosférica de Izaña (CIAI-AEMET)

Omaira E. García Rodríguez

Centro de Investigación Atmosférica de Izaña (CIAI-AEMET)

$M^{a}$ Emma Borges Chinea

Departamento de Ingeniería Química, Universidad de La Laguna (ULL)

Alberto Redondas Marrero

Centro de Investigación Atmosférica de Izaña (CIAI-AEMET)

Juan José Rodríguez Franco

Centro de Investigación Atmosférica de Izaña (CIAI-AEMET)

Rosa Delia García Cabrera

Centro de Investigación Atmosférica de Izaña (CIAI-AEMET),

Grupo de Óptica Atmosférica, Universidad de Valladolid (GOA, UVA)

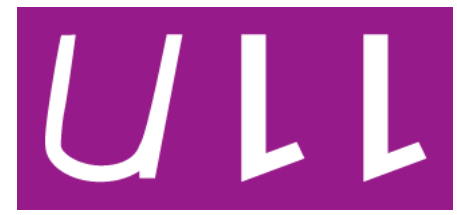




\section{datos \\ abiertos}

Aviso Legal: los contenidos de esta publicación podrán ser reutilizados, citando la fuente y la fecha, en su caso, de la última actualización

\section{Edita:}

(C) Ministerio de Agricultura, Alimentación y Medio Ambiente Agencia Estatal de Meteorología

Madrid, 2012

Catálogo de Publicaciones de la Administración General del Estado: https://cpage.mpr.gob.es

NIPO: 281-12-017-7

https://doi.org/10.31978/281-12-017-7

Agencia Estatal de Meteorología (AEMET)

C/ Leonardo Prieto Castro, 8

28040 Madrid

http://www.aemet.es/

@Aemet_Esp

https://www.facebook.com/AgenciaEstataldeMeteorologia 


\section{Prólogo}

La siguiente Nota Técnica Digital es un resumen del proyecto de Fin de Máster de Ingeniería Química "Intensificación en Investigación en Ingeniería Química, Ambiental y Alimentaria, Año 2011" de la Universidad de La Laguna (ULL), el cual se realizó en el Centro de Investigación Atmosférica de Izaña (CIAI-AEMET) durante el curso 2010 / 2011 en colaboración con la ULL.

El objetivo de este trabajo es analizar la viabilidad del empleo de la radiación solar ultravioleta registrada en el Observatorio Atmosférico de Izaña (OAI), perteneciente al CIAI-AEMET, como fuente de energía para el tratamiento de aguas residuales. Para ello, se estudiarán los fundamentos y principios básicos de operación de los Tratamientos Fotocatalíticos de Oxidación Avanzada (TOAs), y se realizará un análisis detallado de la radiación solar ultravioleta en el OAI. Finalmente, mediante una revisión bibliográfica, se analizará la posibilidad de la aplicación de estas técnicas. 


\section{Contenidos}

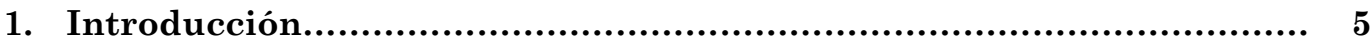

2. Tratamientos de Oxidación Avanzada............................................. 6

2.1. Fotocatálisis Heterogénea con $\mathrm{TiO}_{2} \quad 10$

2.2. Fotocatálisis Homogénea Foto-Fenton $\left(\mathrm{Fe}^{2+/} \mathrm{H}_{2} \mathrm{O}_{2}\right)$

3. Radiación Solar Ultravioleta........................................................... 11

3.1. Espectrofotómetro Brewer 12

3.2. Centro Europeo de Calibraciones Brewer y OAI 14

3.3. Evaluación de la Radiación Solar Ultravioleta en el OAI 15

4. Análisis Comparativo............................................................ 17

4.1. Irradiancia e Irradiación 18

4.2. Energía acumulada por unidad de volumen, Quv 21

4.3. Tiempo de iluminación, $\mathrm{t}_{30 \mathrm{w}} \quad 22$

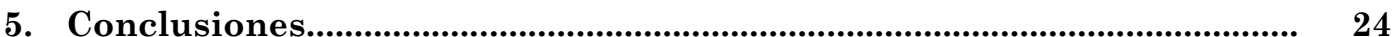

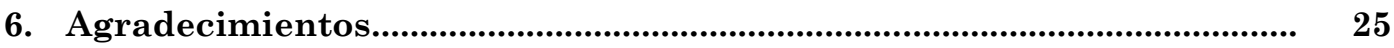

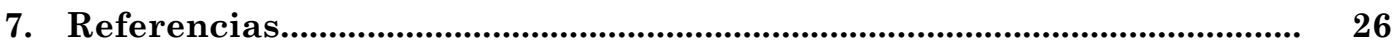




\section{Introducción}

La producción de residuos es una de las características que mejor define la sociedad actual de los países desarrollados. Los vertidos procedentes de la industria y la agricultura constituyen el principal problema, aunque en la contaminación del medio ambiente todos jugamos un papel fundamental. Plaguicidas, fertilizantes, detergentes, fenoles y otros productos químicos son arrojados sin tratamiento directamente a la naturaleza [Acosta, 2009].

De todas las dificultades actuales con las que nos enfrentamos en el medio ambiente, las relativas a la energía y al agua son probablemente las más complicadas de abordar científicamente y las que tienen consecuencias más graves a largo plazo. La falta de agua, que ya es un grave problema mundial, se agudizará durante la primera mitad de este siglo. Si la tendencia actual continúa, dos tercios de la población de la Tierra estará viviendo en áreas con escasez de agua para el año 2025. El peor impacto tendrá lugar en las zonas áridas de los países en desarrollo, en las cuales el promedio disponible por persona será sólo de un $15 \%$ de la disponibilidad per cápita que había en 1950 [Blanco y col., 2009]. El suministro para grandes poblaciones es, por tanto, uno de los mayores retos a afrontar hoy en día.

El problema del agua no puede abordarse de forma efectiva sin tener en cuenta las implicaciones energéticas y el crecimiento poblacional. No hay un desarrollo sostenible en términos de agua y energía sin una fuerte participación de las energías renovables en general y, en particular, de la energía solar, que tiene el mayor potencial de todas ellas. Se necesitan procedimientos innovadores para dar solución a un amplio espectro de problemas, cuya escala de aplicación y complejidad varían notablemente. Por ello, es necesario el estudio detallado de dichos procedimientos y de los factores que determinan su aplicabilidad, como es el caso de la radiación solar ultravioleta, objeto de estudio en este trabajo. 


\section{Tratamientos de Oxidación Avanzada}

El tratamiento de aguas contaminadas de diversos orígenes está en creciente demanda, lo cual se ve reflejado en nuevas legislaciones más estrictas. Este hecho impulsa el desarrollo de nuevas tecnologías para la depuración, puesto que las actuales no permiten, en algunos casos, alcanzar los requerimientos mínimos impuestos por la ley o por el uso posterior que se le va a dar al efluente tratado.

Un ejemplo es el desarrollo de una serie de Tratamientos o Procesos de Oxidación Avanzada (TOAs, POAs), basados en procesos fisicoquímicos capaces de cambiar la estructura química de los contaminantes [EPA, 1998]. Los TOAs generan y hacen uso de especies intermedias, principalmente del radical hidroxilo $\left(\mathrm{OH}^{*}\right)$ el cual es muy efectivo para la oxidación de materia orgánica. En general, el número de contaminantes que han conseguido ser degradados mediante TOAs incluye una gran cantidad de compuestos orgánicos, de entre los cuales cabe destacar los llamados "Contaminantes Emergentes". Estos contaminantes incluyen, entre otros, los fármacos y los productos de uso personal, no regulados aún, pero candidatos a ello [Belgiorno y col., 2007]. Pero los TOAs no sólo son capaces de degradar compuestos orgánicos, sino que permiten realizar transformaciones en contaminantes tóxicos poco susceptibles a la oxidación, como iones metálicos o compuestos halogenados. Asimismo, el gran potencial de estos tratamientos se ve incrementado por la existencia de diversas formas de producir los radicales hidroxilo, facilitando la aplicación de estas tecnologías.

Las Ventajas y Características más positivas de los TOAs, con respecto a los métodos convencionales, son las siguientes:

- Transforman químicamente el contaminante.

- Destruyen o mineralizan completamente el contaminante, hasta dióxido de carbono e iones como cloruros.

- Normalmente no se generan lodos.

- Muy útiles para contaminantes refractarios que resisten otros métodos de tratamiento.

- Tratan contaminantes a muy baja concentración. 
- Tienen una reactividad no selectiva.

- No se forman subproductos de reacción o lo hacen en muy baja concentración.

- Disminuyen la concentración de compuestos formados por pretratamientos alternativos como la desinfección.

- Mejoran las propiedades organolépticas del agua tratada.

- Permiten transformar contaminantes refractarios en productos tratables por métodos más económicos.

- Eliminan efectos sobre la salud de desinfectantes y oxidantes residuales como el cloro.

No obstante, presentan también una serie de Desventajas como:

- Elevado coste por el uso de reactivos caros.

- Restricción de uso en función del grado de contaminación del agua (Demanda Química de Oxígeno, DQO) por razones económicas y de índole cinético.

A continuación se muestra un resumen de estas nuevas técnicas de oxidación (tabla 2.1), donde se ha diferenciado entre procesos que hacen uso de energía radiante (procesos fotoquímicos), de los que no (procesos no fotoquímicos).

Tabla 2.1 : Resumen de Tratamientos de Oxidación Avanzada.

\begin{tabular}{|c|c|}
\hline $\begin{array}{l}\text { Procesos NO } \\
\text { Fotoquímicos }\end{array}$ & $\begin{array}{l}\text { - Descarga electrohidráulica - Ultrasonido } \\
\text { - Oxidación electroquímica } \\
\text { - Ozonización con peróxido de hidrógeno }\left(\mathrm{O}_{3} / \mathrm{H}_{2} \mathrm{O}_{2}\right) \\
\text { - Ozonización en medio alcalino }\left(\mathrm{O}_{3} / \mathrm{OH}^{-}\right) \\
\text {- Plasma no térmico } \\
\text { - Procesos Fenton }\left(\mathrm{Fe}^{2+} / \mathrm{H}_{2} \mathrm{O}_{2}\right) \text { y relacionados } \\
\text { - Radiólisis y tratamiento con haces de electrones }\end{array}$ \\
\hline $\begin{array}{c}\text { Procesos } \\
\text { Fotoquímicos }\end{array}$ & $\begin{array}{l}\text { - Fotocatálisis heterogénea } \\
\text { - Foto-Fenton y relacionadas } \\
\text { - Fotólisis del agua en el ultravioleta de vacío (UVV) } \\
\text { - Oxidación en agua sub y supercrítica } \\
\text { - Procesos fotoquímicos } \\
\text { - }{\mathrm{UV} / \mathrm{O}_{3}} \\
\text { - } \mathrm{UV} / \mathrm{H}_{2} \mathrm{O}_{2}\end{array}$ \\
\hline
\end{tabular}


De entre estos tratamientos, este trabajo se centra en aquellos procesos fotoquímicos que hacen uso de la energía solar para sus transformaciones [Malato y col., 2009], por su importante valor medioambiental, ya que constituyen claros ejemplos de tecnologías sostenibles.

Los métodos fotoquímicos para la degradación de contaminantes disueltos en agua se basan en proporcionar energía a los compuestos químicos, la cual es absorbida por las distintas moléculas para alcanzar estados excitados el tiempo necesario para experimentar reacciones. La energía radiante es absorbida por las moléculas en forma de unidades cuantizadas denominadas fotones, los cuales han de contener la energía necesaria para excitar electrones específicos y formar radicales libres, dando lugar a una serie de reacciones en cadena. Los métodos basados en la radiación electromagnética utilizan normalmente radiación ultravioleta (UV, 4-400 nm) debido a la mayor energía de sus fotones, tal como indica la ecuación de Plank:

$$
E_{\lambda}=\frac{h \cdot c}{\lambda}
$$

donde

(Ecuación 2.1)

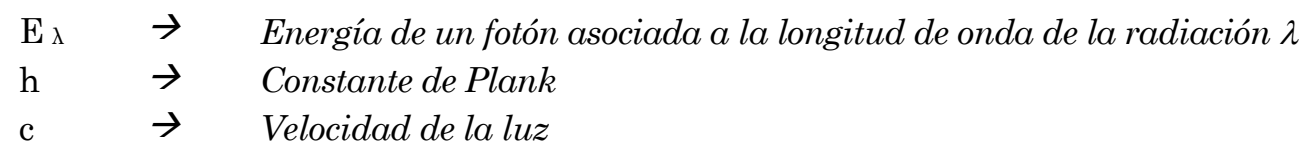

Si en estos procesos fotoquímicos participa un catalizador, hablamos de Fotocatálisis [Mills y col., 1997]. La generación artificial de los fotones requeridos para la activación fotocatalítica es la principal fuente de costes durante la operación de las plantas de tratamiento de aguas residuales. De ahí, la importancia de buscar fuentes alternativas de energía y desarrollar tecnologías sostenibles. En este contexto se enmarca la fotocatálisis solar para la descontaminación de aguas, una de las aplicaciones más exitosas de la fotoquímica solar, lo cual es corroborado con la variedad de proyectos e instalaciones que están actualmente en operación [Blanco y col., 2009]. La capacidad de la energía solar UV de fotodegradar, en condiciones catalíticas, a un vasto número de contaminantes ha sido ampliamente demostrada en la literatura [Shawaqfeh y col., 2010; Radjenovic y col., 2010; Farlas y col., 2010, Michael y col., 2010; Oyama y col., 2004, etc].

La fotocatálisis solar puede ser de dos tipos:

- Heterogénea: un sólido semiconductor forma una suspensión estable bajo irradiación para estimular una reacción en la interfase sólido / líquido o sólido / gas.

- Homogénea: el catalizador está disuelto en el agua que contiene los contaminantes a tratar, también disueltos, por lo que todos los elementos que intervienen en el proceso se encuentran en la misma fase.

En ambos casos, por definición, el catalizador debe de poder ser reutilizado después de actuar en el sistema redox sin sufrir cambios significativos. 
La eficacia de una reacción fotocatalítica depende de ciertos factores que gobiernan la cinética de la fotocatálisis como, por ejemplo, la concentración inicial de reactante, radiación solar ultravioleta, masa de catalizador, $\mathrm{pH}$, temperatura, etc. No obstante, para todos los tipos de reacciones fotocatalíticas se ha observado que el índice de cambio de la reacción es directamente proporcional al flujo radiante, lo cual confirma la naturaleza fotoinducida de la activación del proceso fotocatalítico, con la participación de la carga eléctrica fotoinducida (electrones y huecos) en el mecanismo de reacción [Malato y col., 2009]. Además, en determinados estudios se observa que la degradación de contaminantes incrementa con el aumento de la irradiancia solar UV, mostrando una dependencia lineal con la intensidad de la luz en el rango de 20 a $30 \mathrm{~W} / \mathrm{m}^{2}$ [Nogueira y col., 1996].

El responsable de la variación de la velocidad de la reacción en función de la longitud de onda es el espectro de absorción del catalizador, el cual fija el umbral correspondiente en su Banda de Energía. Así, en la figura 2.1 se muestran, a modo de ejemplo, los espectros de absorción de una suspensión de $\mathrm{TiO}_{2}$ y de una disolución de $\mathrm{Fe}_{2} \mathrm{SO}_{4}$. Éstos definen los procesos fotocatalíticos heterogéneos y homogéneos más efectivos respectivamente, los cuales se describen brevemente a continuación.

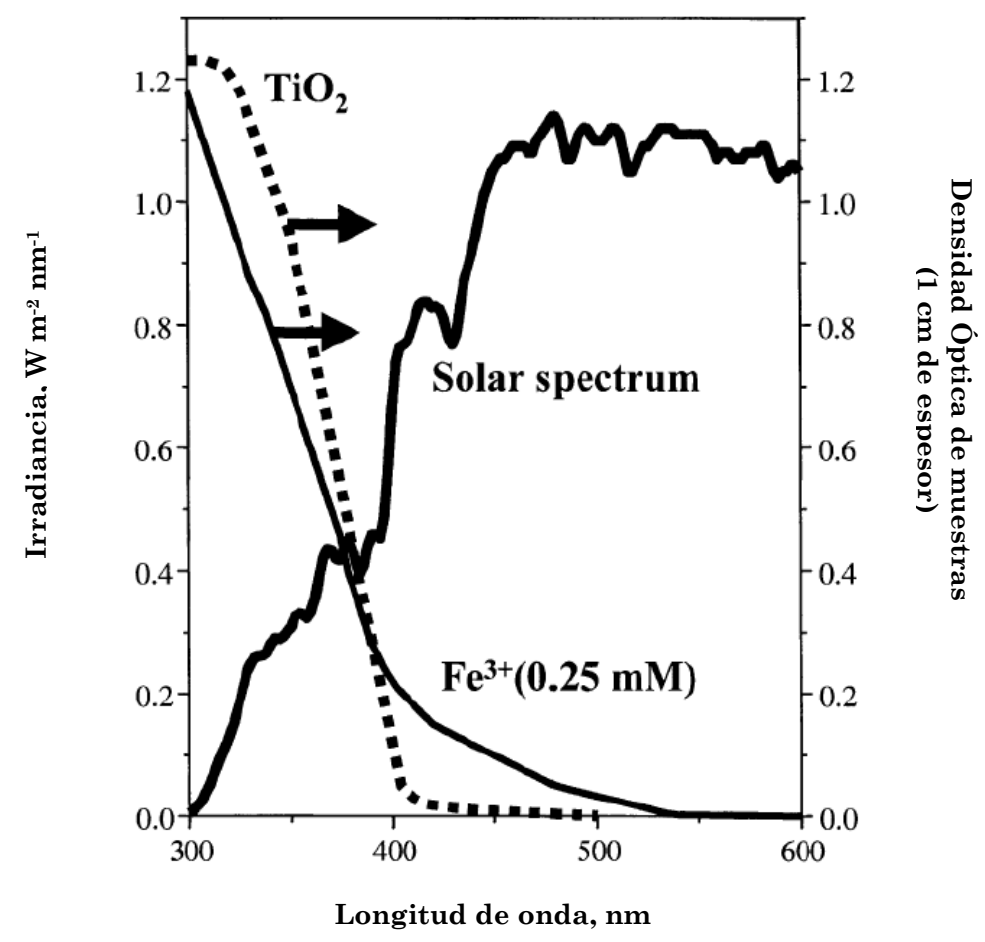

Figura 2.1 : Espectro de absorción de la irradiancia solar (línea continua gruesa) comparado con los espectros de absorción de una disolución de $\mathrm{Fe}_{2} \mathrm{SO}_{4}(0,25 \mathrm{mM}$ de $\mathrm{Fe}$, línea continua fina) y una suspensión de $\mathrm{TiO}_{2}(50 \mathrm{mg} / \mathrm{l}$, línea punteada) [Malato y col., 2002]. 


\subsection{Fotocatálisis heterogénea con $\mathrm{TiO}_{2}$}

La fotocatálisis heterogénea, a diferencia de la mayoría de los procesos fotoquímicos, no es selectiva y puede emplearse para tratar mezclas complejas de contaminantes. Para una catálisis heterogénea básica la totalidad del proceso se puede descomponer en cinco pasos [Herrmann, 1999], ocurriendo la reacción fotocatalítica en el tercero:

1) Transferencia de los reactivos en el fluido a la superficie del catalizador.

2) Adsorción de, al menos, uno de los reactantes.

3) Reacción en la fase adsorbente.

4) Desorción de los productos.

5) Eliminación de los productos de la región interfase.

Han sido varios los materiales semiconductores probados bajo condiciones comparables para la degradación de los mismos compuestos y el $\mathrm{TiO}_{2}$ ha demostrado, en general, ser el más efectivo. Las ventajas más significativas de este compuesto son su fuerte resistencia a la ruptura química y a la fotocorrosión, su bajo coste y su seguridad. Además, el $\mathrm{TiO}_{2}$ interacciona eficientemente con la radiación solar UV, ya que la separación energética entre sus bandas de valencia y conducción puede ser superada por la energía solar en el rango 300-390 nm.

Puesto que su actividad catalítica puede verse potenciada por varios factores, numerosos compuestos se han empleado para mejorar la eficiencia del $\mathrm{TiO}_{2}$ como fotocatalizador (ceniza volcánica, fibra óptica de cuarzo, fibra de cristal, lana, etc), [Pozzo y col., 1997; Borges y col., 2008; Esparza y col., 2010; Esparza y col., 2011].

\subsection{Fotocatálisis homogénea Foto-Fenton $\left(\mathrm{Fe}^{2+} / \mathrm{H}_{2} \mathrm{O}_{2}\right)$}

El proceso Foto-Fenton, basado en la generación de radicales hidroxilo en presencia de peróxido de hidrógeno e iones ferrosos, parece, a priori, el más indicado de todos los TOAs para ser llevado a cabo mediante radiación solar, ya que el hidróxido de hierro es soluble en agua y los complejos orgánicos ácidos de hierro absorben marcadamente en el UV e incluso parte del espectro visible (VIS). Esto da lugar a que el proceso sea muy eficiente para la generación de radicales en los procesos oxidativos. En presencia de radiación solar el proceso se convierte en fotocatalítico, ya que el ión $\mathrm{Fe}^{2+}$ oxidado a $\mathrm{Fe}^{3+}$ en la reacción de Fenton se reduce de nuevo a $\mathrm{Fe}^{2+}$ por acción de la radiación. Una mejora para los procesos Foto-Fenton es la introducción de ferrioxalato. El sistema UV-VIS/Ferrioxalato/ $\mathrm{H}_{2} \mathrm{O}_{2}$ ha demostrado ser más eficiente que el Foto-Fenton para la eliminación de contaminantes orgánicos [Andreozzi y col., 1999].

Una de las principales ventajas del proceso Foto-Fenton frente al $\mathrm{TiO}_{2}$ es su mayor sensibilidad a la radiación solar, lo que supone un mayor aprovechamiento de la misma. Asimismo, la radiación solar presenta una mayor capacidad de penetración al tratarse de una disolución, mientras que la transferencia de la radiación en una suspensión de $\mathrm{TiO}_{2}$ es muy compleja y a menudo se convierte en el principal factor limitante al diseñar reactores fotocatalíticos. Finalmente, como la solución es homogénea, el contacto entre el contaminante y el agente oxidante es mayor. 


\section{Radiación Solar Ultravioleta}

La radiación solar se define como el flujo de energía que recibimos del Sol en forma de ondas electromagnéticas de diferentes frecuencias. Aproximadamente, la mitad de las que recibimos pueden ser detectadas por el ojo humano, constituyendo lo que se conoce como luz visible (VIS, entre 400 y $700 \mathrm{~nm}$ ). De la otra mitad, la mayoría se sitúa en la parte infrarroja del espectro (IR, entre 760 y $10.000 \mathrm{~nm}$ ) y una pequeña parte en la región ultravioleta (UV, entre 4 y $400 \mathrm{~nm}$ ).

Entre las múltiples aplicaciones de la energía solar se encuentran: su aprovechamiento como luz directa, como fuente de calor y en la generación de electricidad. La primera de estas aplicaciones, como fuente de energía para procesos fotocatalíticos, es el objetivo de este trabajo y en la que se centran la mayoría de los estudios realizados sobre TOAs. Como se comentó anteriormente, son numerosos los autores que han documentado los buenos resultados del empleo de la radiación solar, en especial el rango UV, para la degradación de contaminantes [Shawaqfeh y col., 2010; Radjenovic y col., 2010; Farlas y col., 2010, Michael y col., 2010; Oyama y col., 2004, etc].

La energía solar UV con longitudes de onda por debajo de $300 \mathrm{~nm}$ (UVC y parte del UVB) es absorbida completamente por los gases de la atmósfera terrestre (fundamentalmente por el ozono), mientras que la mayoría de la radiación solar UV entre 300 y $400 \mathrm{~nm}$ alcanza la superficie terrestre y se convierte en calor [Zerefos y col., 1997]. La medida y seguimiento de esta porción de radiación UV se ha incrementado sustancialmente en los últimos 20 años, tanto por el deseo de mitigar sus potenciales efectos nocivos sobre la salud humana y la biosfera, así como por el papel que juega en el cambio climático.

Son dos las formas en que puede cuantificarse la radiación UV que alcanza la superficie terrestre: por medida directa, mediante el uso de instrumentación adecuada, e indirecta, a través de la simulación de la transferencia radiativa en la atmósfera mediante modelos numéricos. Ambos métodos tienen sus ventajas y sus inconvenientes, pero el que emplearemos en el presente trabajo es la medida directa ya que la simulación de la energía UV depende de parámetros auxiliares que no siempre están disponibles. 
Existen numerosos sistemas de medida de la radiación solar UV, si bien el diseño óptimo, en términos de calidad de los datos, es un doble monocromador con un tubo fotomultiplicador (PMT) como detector y la óptica de entrada adecuada [Webb, 2003]. Estas características describen al espectrofotómetro Brewer, ampliamente utilizado por la comunidad científica internacional y empleado en este trabajo para la medida de la radiación solar ultravioleta, el cual se describe a continuación.

\subsection{Espectrofotómetro Brewer}

El equipo Brewer fue desarrollado a principios de los años 80's para medir a nivel del suelo la intensidad de la radiación solar, global y directa, en la región espectral UV y/o VIS [Kerr et al., 1981]. Estas medidas permiten derivar la concentración de aquellos gases atmosféricos que presenten una fuerte absorción en este rango espectral, como el ozono o el dióxido de azufre, así como la monitorización continua de la radiación UV. En la actualidad este equipo se encuentra operativo en más de 40 países, siendo más de 200 los que realizan medidas con regularidad [Savastiouk, 2006].

El sistema Brewer se compone de: un espectrofotómetro que mide la radiación solar global y directa de manera espectral (consta de un sistema de monocromación y un detector), un seguidor solar (compuesto por un trípode y un tracker), el equipo informático de control de funcionamiento y un software de registro de datos. El Brewer está diseñado para su uso continuado al aire libre, por lo que el espectrómetro se encuentra en el interior de una resistente carcasa que protege los componentes internos. Por ello, funciona de forma fiable y precisa en una amplia gama de condiciones de temperatura y humedad [SCI-TEC Instruments Inc.,1998]. La figura 3.2 muestra un esquema de los distintos componentes del sistema Brewer, mientras que la tabla 3.1 resume sus principales especificaciones técnicas.

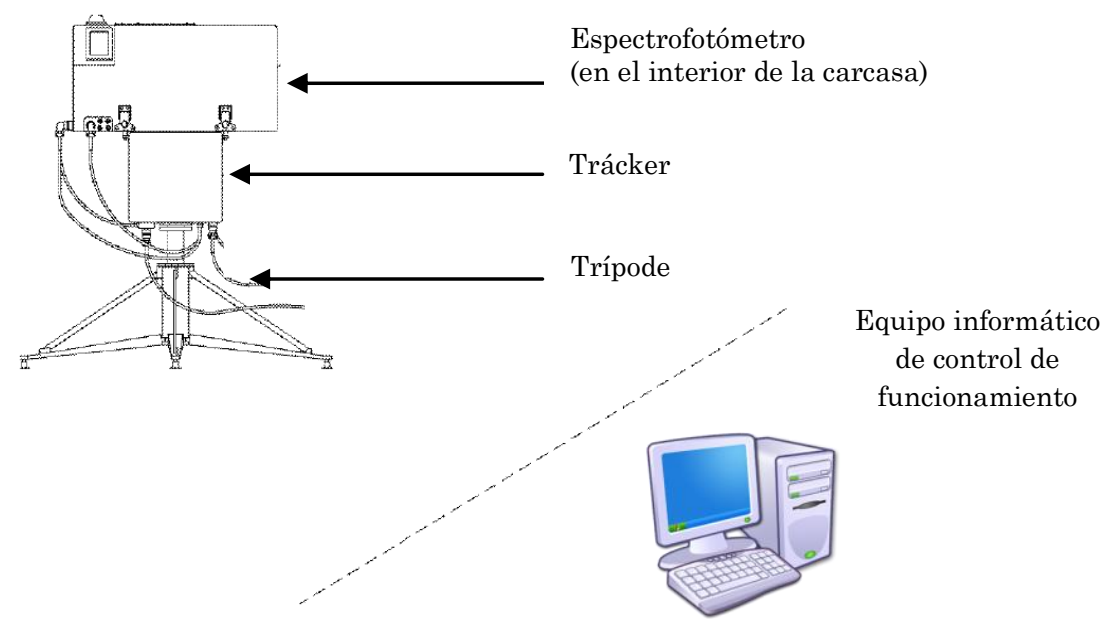

Figura 3.2 : Esquema del sistema Brewer (Imagen modificada de SCI-TEC Instruments Inc,1998). 
Tabla 3.1 : Especificaciones técnicas del Brewer.

\begin{tabular}{|c|c|}
\hline Longitudes de onda ultravioleta & $\begin{array}{l}\text { Longitudes de onda del "ozono": } 303,2 \text { (rendija } \\
\mathrm{Hg} \text { - } 306,3-310,1-313,5-316,8-320,1 \mathrm{~nm}\end{array}$ \\
\hline Calibración de mercurio & $302,15 \mathrm{~nm}$ \\
\hline Resolución & $0,6 \mathrm{~nm}$ en UV \\
\hline Estabilidad & $\pm 0,01 \mathrm{~nm}$ (en todo el rango de temperaturas) \\
\hline Precisión & $0,006 \pm 0,002 \mathrm{~nm}$ \\
\hline Rango de medida & $286,5 \mathrm{~nm}$ a $363,0 \mathrm{~nm}$ \\
\hline Ciclos de la máscara de la rendija de salida & $0,12 \mathrm{seg} /$ rendija, $1,6 \mathrm{seg}$ para el ciclo completo \\
\hline Exactitud de la medida de ozono & $\pm 1 \%$ (para el ozono total a sol directo) \\
\hline Rango de temperatura ambiente de operación & $\begin{array}{l}0^{\circ} \mathrm{C} \text { a }+40^{\circ} \mathrm{C} \text { (sin calefacción) } \\
-20^{\circ} \mathrm{C} \text { a }+40^{\circ} \mathrm{C} \text { (con opción de calefacción) } \\
-50^{\circ} \mathrm{C} \text { a }+40^{\circ} \mathrm{C} \text { (con el kit completo de tiempo frío) }\end{array}$ \\
\hline Dimensiones físicas (carcasa externa) & $\begin{array}{l}\text { Peso: } \quad 34 \mathrm{~kg}(75 \mathrm{lbs}) \\
\text { Tamaño : } 70 \times 46 \times 34 \mathrm{~cm}\end{array}$ \\
\hline $\begin{array}{l}\text { Requerimientos energéticos del Brewer y el } \\
\text { Tracker }\end{array}$ & $\begin{array}{l}\text { 3A @ } 80 \text { a } 140 \text { VAC (con opción de calefacción) } \\
1,5 \mathrm{~A} @ 160 \text { a } 264 \text { VAC } \\
47 \text { a } 440 \mathrm{~Hz}\end{array}$ \\
\hline
\end{tabular}

Tradicionalmente, la radiación solar se define en función de sus componentes: directa (aquella que llega directamente del Sol sin haber sufrido cambios en su dirección) y difusa (aquella que procede de cualquier dirección excepto del Sol, como resultado de los múltiples procesos de dispersión y absorción que sufre en la atmósfera). La suma de estas dos componentes recibe el nombre de radiación global. Como se comentó anteriormente, el equipo Brewer permite medir tanto la componente directa como la radiación global, para lo cual tiene fundamentalmente dos posibilidades de funcionamiento: una rápida, empleada para la determinación del contenido total de ozono midiendo la radiación UV directa, en ciclos de cinco longitudes de onda fijas mientras la red de difracción permanece inmóvil; y otra lenta para la medida de la radiación global UV, en la que la red de difracción se mueve realizando dos barridos (arriba y abajo) entre $290 \mathrm{~nm}$ y $363 \mathrm{~nm}$. Para ello el subconjunto óptico capta la luz a través de la rendija de entrada y la dispersa en un espectro de alta calidad a lo largo del plano de la rendija de salida, donde hay una máscara cilíndrica que filtra el paso de luz (asociada a una determinada longitud de onda).

Son varias las fuentes de error e incertidumbre asociadas a la medida de la irradiancia global espectral UV con este instrumento. Dichas fuentes incluyen el "stray light" (luz externa) y el ruido, la estabilidad y calibración del equipo, el desalineamiento en longitud de onda, la resolución espectral y errores de tiempo. El error asociado con la respuesta coseno (respuesta del instrumento con el ángulo de incidencia de los rayos solares [Bais y col., 1998]) puede variar entre un 3 y un $10 \%$ para ángulos cenitales mayores de $60^{\circ}$, disminuyendo este error para ángulos cenitales menores. Este error se corrige con una adecuada caracterización del equipo [Redondas, 2000]. La dependencia de la temperatura también pueden desempeñar un papel significativo en la determinación del error total [Hansen, 2009]. Asumiendo que estas fuentes de error se hallan acotadas, varios autores estiman la incertidumbre asociada a las medidas de radiación espectral UV en $\pm 3 \%$ ( $1 \sigma)$ [Fioletov y col., 2001; Gröbner y col., 2004; http://www.woudc.org]. 


\subsection{Centro Europeo de Calibraciones Brewer y OAI}

Los datos empleados para el análisis de la radiación solar ultravioleta en este trabajo fueron tomados con el espectrofotómetro Brewer\#157, patrón absoluto europeo y uno de los tres equipos que conforman la triada de referencia (Brewer\#157, Brewer\#183 y Brewer\#185) del Centro Europeo de Calibraciones Brewer (Regional Brewer Calibration Center - Europe, RBCC-E). Dicho organismo fue creado en 2003 por recomendación de la Organización Meteorológica Mundial (OMM) en el Centro de Investigación Atmosférica de Izaña (CIAI, www.izana.org), dependiente de la Agencia Estatal de Meteorología (www.aemet.es). Su finalidad es la colaboración en la validación de satélites y en la calibración de los espectrofotómetros Brewer europeos y del norte de África. El programa de medida de Ozono/UV del CIAI, donde se desarrolla el trabajo del RBCC, se fundamenta en tres objetivos principales:

1) Medición continua, y a largo plazo, del ozono y de la radiación UV conforme a las más altas exigencias de precisión y de calidad.

2) Estudio y vigilancia del ozono y la radiación UV en la región subtropical.

3) Investigación y desarrollo de nuevas técnicas instrumentales y/o metodológicas, encaminadas a mejorar las medidas de ozono.

La tríada de espectrofotómetros se encuentra ubicada en el Observatorio Atmosférico de Izaña (OAI), uno de los dos observatorios del CIAI. El OAI, situado en la isla de Tenerife (Islas Canarias, figura 3.2), se encuentra a $2367 \mathrm{~m}$ sobre el nivel del mar, en la troposfera libre, por encima de una capa de inversión cuasi-permanente debida al movimiento descendente de aire asociado al anticiclón de las Azores. El clima en la zona de la estación es extremadamente seco durante la mayor parte del año y la insolación es muy alta, especialmente durante el verano, registrándose los valores más altos de toda España con una media de 3448,5 horas/año (www.izana.org). La elección del OAI para emplazar el RBCC-E es debida fundamentalmente a dos factores:

1) Las condiciones del cielo en el OAI, consecuencia de la situación subtropical del archipiélago, la cual se caracteriza por una concentración relativamente baja de ozono, con escasa variabilidad diaria y anual. Esto hace que la calibración solar absoluta de los espectrofotómetros sea óptima desde un punto de vista atmosférico. Otro factor decisivo es la altitud, proporcionando una gran transparencia del cielo con magníficas condiciones para la obtención de medidas radiométricas solares.

2) La experiencia científico-técnica del personal en la operación y calibración de espectrofotómetros Brewer y en la organización de campañas internacionales de calibración. 


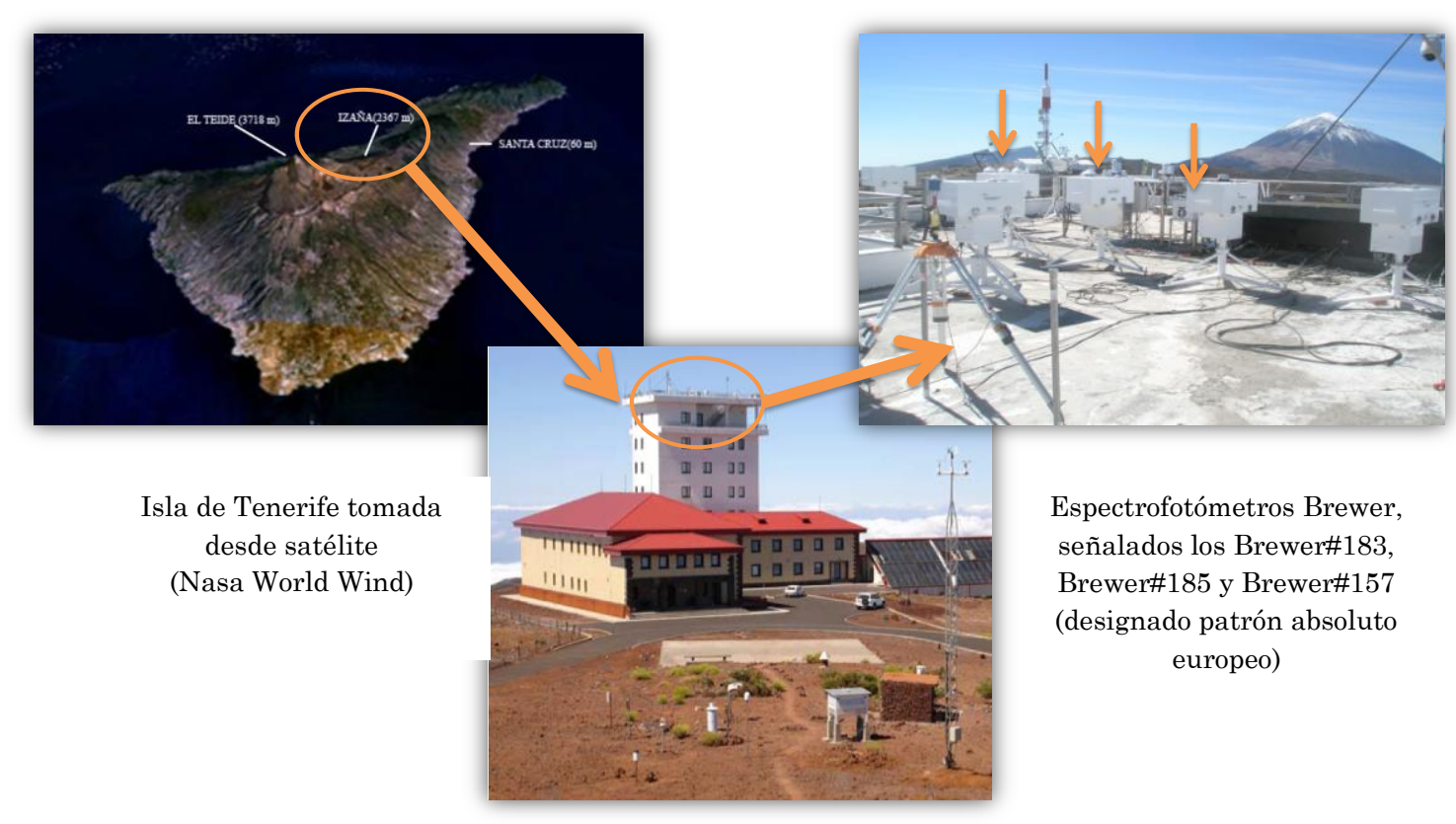

Observatorio Atmosférico de Izaña (CIAI) [García, 2009]

Figura3.2 : Localización de los espectrofotómetros Brewer en el Observatorio Atmosférico de Izaña.

Toda la actividad llevada a cabo por el RBCC-E es difundida públicamente a través de su web (http://www.rbcc-e.org). Además, a través del portal Iberonesia (http://www.iberonesia.net/brewer) es posible realizar consultas de la base de datos en la que diariamente se vuelcan los proporcionados por la referencia europea. No sólo se pueden consultar datos de ozono total en columna y radiación UV medidos por la triada de referencia, sino que también son accesibles datos provenientes de espectrofotómetros Brewer del resto de España, Marruecos y Portugal. Asimismo, es posible chequear el estado y la calibración vigente de cada instrumento.

\subsection{Evaluación de la Radiación Solar UV en el OAI}

Los espectrofotómetros de alta resolución, como es el caso del Brewer, proporcionan medidas de alta calidad de la radiación solar UV. Sin embargo para ello requieren una atención continuada de personal especializado para su mantenimiento, así como frecuentes chequeos y calibraciones.

La calibración del equipo para la medida ultravioleta se basa en el control de dos parámetros fundamentales: longitud de onda e irradiancia. La calibración en longitud de onda se realiza mediante los "Test de Dispersión" (figura 3.3, derecha) utilizando líneas espectrales de lámparas de descarga que poseen líneas en el rango ultravioleta como son las de $\mathrm{Hg}, \mathrm{Cd}$ y Zn. Esta asignación se mantiene mediante el test de lámpara de mercurio [Gröbner y col., 1998]. La calibración absoluta en irradiancia se realiza con una lámpara de 1000W (figura 3.3, izquierda), ya que con esta potencia se obtienen irradiancias 
comparables a la solar. Estas lámparas están referenciadas a patrones internacionales y requieren de instrumentación y operaciones complejas. La calibración del equipo se comprueba mediante medidas quincenales con lámparas de $200 \mathrm{~W}$.
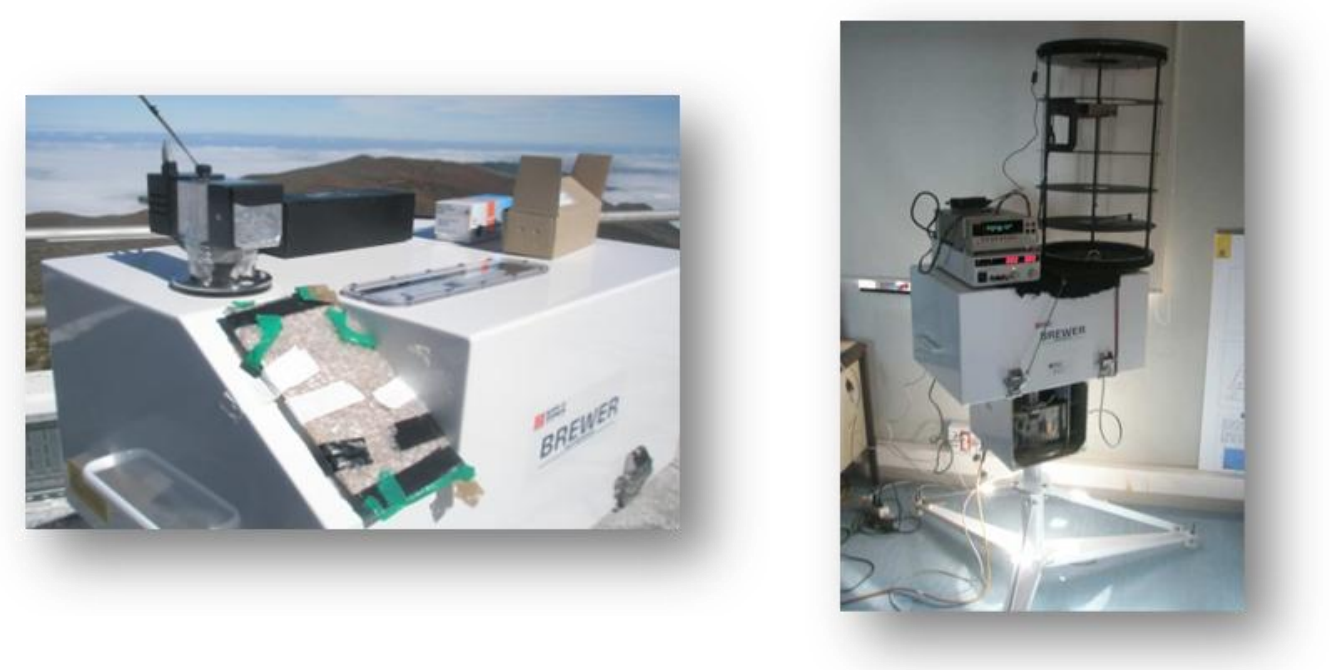

Figura 3.3 : Espectrofotómetro Brewer durante la realización de los test de dispersión (izquierda) y durante la calibración con lámparas de 1000W en el SetUp portátil (derecha).

Una vez calibradas y depuradas las medidas del equipo se obtienen los espectros de irradiancia global solar (Irradiancia $(\lambda, \mathrm{t})$ ), como el que se muestra en la figura 3.4 a modo de ejemplo.

\section{1 de Enero de 2010}

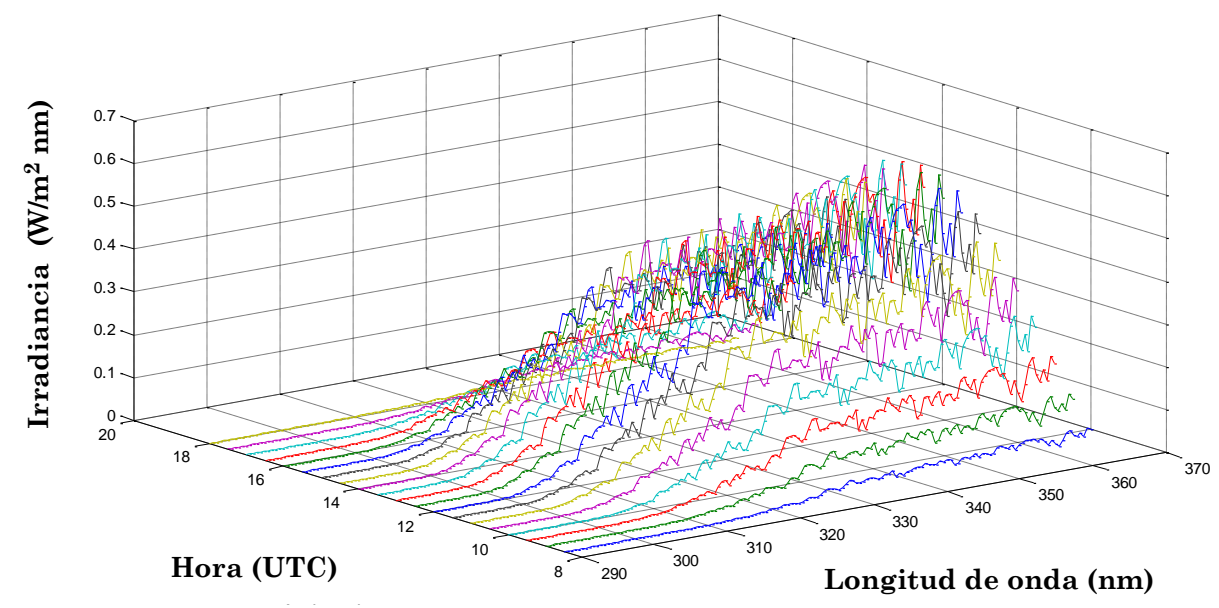

Figura 3.4 : Evolución diaria de la irradiancia global espectral $\left(\mathrm{W} / \mathrm{m}^{2} \mathrm{~nm}\right)$ para el 1 de Enero de 2010. 


\section{Análisis Comparativo}

Para poder implementar las tecnologías fotocatalíticas para el tratamiento de aguas residuales, comentadas en la sección 2, es necesario conocer y caracterizar la distribución de radiación UV del emplazamiento en el que situaría el sistema de depuración. Los valores de irradiancia solar incidente dependen del lugar, hora del día, época del año y estado del cielo. Por tanto, es muy importante la correcta ubicación del espectrofotómetro, puesto que cuánto más cerca esté de la zona de estudio más fiables serán nuestros resultados sobre la viabilidad de una posible planta piloto. Así, en este trabajo, se ha analizado la radiación solar ultravioleta en el Observatorio Atmosférico de Izaña (OAI) en el año 2010 para evaluar la posibilidad de usar estas técnicas en dicho emplazamiento.

Las medidas de radiación global UV en el OAI han sido evaluadas en términos de irradiancia e irradiación, energía acumulada por unidad de volumen (Quv) y tiempo de iluminación $\left(\mathrm{t}_{30 \mathrm{~W}}\right)$ para poder ser comparadas con la literatura. Se han estimado valores diarios de estas magnitudes, considerando así el número total de horas de iluminación diarias (del orto al ocaso) y no únicamente un periodo de tiempo determinado. Como se ha comentado previamente, los espectrómetros Brewers en el OAI están destinados tanto a la medida de radiación directa como global, por lo que estas rutinas de muestreo se van alternando durante el día. Esto provoca que la frecuencia de las medidas de radiación global UV no sea uniforme, además de poder existir lagunas de datos horarios, lo que afecta a la integración de los valores diarios. Para evitar la posible subestimación de los valores diarios obtenidos a partir de las medidas experimentales, se han empleado en este trabajo los valores diarios estimados del ajuste de la evolución diaria de la irradiancia global a una función gaussiana. Se tomaron como días válidos aquellos donde el coeficiente de determinación de dicho ajuste fuera superior al 98,5\% $\left(R^{2}\right)$. Esta condición solo se cumple en ausencia de nubosidad, por lo que el estudio ha sido realizado considerando únicamente días despejados. El porcentaje de datos con los que contamos en estas condiciones es del 89\% del total de medidas realizadas por el equipo en 2010 (164 días). Para comprobar la bondad de esta aproximación los valores diarios obtenidos mediante esta metodología se compararon con los valores diarios en condiciones de cielo totalmente despejado, facilitados por la estación radiométrica que la Baseline Surface Radiation Network (BSRN, 
http://www.izana.org/bsrn iza) posee en el OAI: la diferencia de la media anual entre los dos conjuntos de datos no supera el $1 \%$.

Al tratarse de medidas experimentales de radiación solar UV, el rango espectral considerado depende de la instrumentación empleada. Así, en el OAI se emplea un espectrómetro Brewer, midiendo de 290 a $366 \mathrm{~nm}$, mientras que en la literatura normalmente se registra con piranómetros de banda ancha, que proporcionan todo el rango UV integrado de 290 a $400 \mathrm{~nm}$. Esto supone un déficit en la radiación integrada obtenida en el OAI, lo que se compensa como veremos a continuación por el aumento de radiación derivado de la altitud y latitud en la que se sitúa el OAI, así como por las condiciones de cielo despejado impuestas. Aunque nuestro estudio asume condiciones de cielo despejado, también en cielos nublados se produce degradación, pero en menor medida.

\subsection{Irradiancia e Irradiación}

La radiación solar ultravioleta es normalmente expresada como una "Irradiancia" (potencia incidente sobre una superficie de una unidad de área, ecuación 4.1) en W/m².

$$
\operatorname{Irradiancia} \text { global }(t)=\int_{290}^{363} \mathrm{~nm} \operatorname{Irradiancia} \text { espectral }(\lambda, \mathrm{t}) \cdot \mathrm{d} \lambda
$$

(Ecuación 4.1)

donde la irradiancia espectral corresponde al flujo energético recibido a cada longitud de onda en una superficie perpendicular a la radiación incidente y se mide en $\mathrm{W} / \mathrm{m}^{2} \mathrm{~nm}$. A partir de esta definición, la irradiancia media diaria se obtiene como la integral entre el orto y el ocaso, divida por el número total de horas de iluminación.

La evolución anual de los valores medios mensuales de irradiancia global UV durante el 2010 en el OAI puede verse en la figura 4.1, donde observamos, como era de esperar, que los valores máximos se registran en los meses de verano y los mínimos en los meses de invierno. Este comportamiento es debido a la evolución anual de la posición relativa de la Tierra y el Sol, que definen la variación anual de la elevación solar. A mayor elevación solar el recorrido que realizan los rayos del Sol en la atmósfera disminuye, sufriendo menos procesos de absorción y dispersión y resultando, por tanto, valores máximos de radiación en superficie. Así, se registra un valor mínimo mensual $( \pm 1 \sigma, \sigma$ desviación estándar) en diciembre de 10,9 $\pm 0,4 \mathrm{~W} / \mathrm{m}^{2}$, mientras que el máximo anual casi duplica este valor en junio, 19,4 $\pm 0,8 \mathrm{~W} / \mathrm{m}^{2}$. La media anual durante 2010 es de $15,4 \pm 3,3 \mathrm{~W} / \mathrm{m}^{2}$, la cual no difiere sustancialmente de la media anual, por ejemplo, en la Plataforma Solar de Almería (PSA), $18 \mathrm{~W} / \mathrm{m}^{2}$. La PSA es el principal centro de investigación, desarrollo y ensayos de Europa dedicado a las tecnologías solares de concentración (http://www.psa.es/webesp/index.php), en el cual se han desarrollado la mayoría de los estudios referenciados en este trabajo. 


\section{Irradiancia Media Mensual 2010}

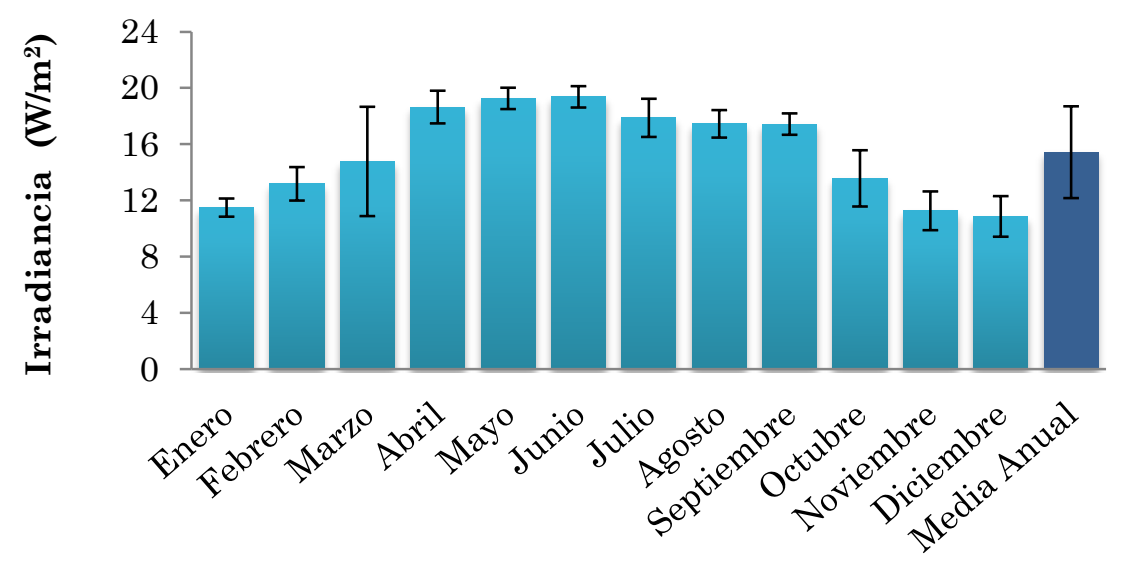

Figura 4.1 : Valores medios mensuales de irradiancia global UV $\left(\mathrm{W} / \mathrm{m}^{2}\right)$ en el OAI para el año 2010. Las barras de error indican $\pm 1 \sigma$ ( $\sigma$, desviación estándar).

Aunque la irradiancia es la forma más común de expresar los datos de radiación solar, es la menos empleada para la descripción de experimentos prácticos llevados a cabo para la destrucción de contaminantes. La irradiancia solar depende de varios factores (latitud, altitud, hora del día, duración de la medida, etc.), por lo que es necesaria una completa descripción de las condiciones de medida para realizar una comparación directa con los resultados de la literatura. Algunos autores hacen referencia a esta magnitud, pero sin especificar el rango de longitudes de onda que integra la medida, rango temporal de la misma o el equipo empleado. Por ello, se introducen en la literatura otras magnitudes derivadas de la irradiancia, como la "Exposición Radiante o Irradiación" (energía incidente sobre una superficie de área unidad durante un periodo de tiempo, ecuación 4.2) en $\mathrm{J} / \mathrm{m}^{2} \mathrm{u}$ otros términos, como la "Energía acumulada por unidad de volumen, Quv" o el "Tiempo de iluminación, t30w”, conceptos descritos en las siguientes secciones.

$$
\text { Irradiación }=\int_{\text {orto }}^{\text {ocaso }} \int_{290}^{363 \mathrm{~nm}} \operatorname{Irradiancia} \text { espectral }(\lambda, \mathrm{t}) \cdot \mathrm{d} \lambda \cdot \mathrm{dt}
$$

(Ecuación 4.2)

Como se comentaba anteriormente, son pocos los artículos que hacen referencia a datos de irradiancia para describir sus resultados; algunos de ellos se detallan en la tabla 4.1, en la que se incluye también los valores de irradiación obtenidos considerando la irradiancia media y el tiempo de iluminación proporcionados. 
Tabla 4.1 : Estudios para la irradiancia global UV. También se proporcionan los valores de irradiación para el tiempo de iluminación proporcionado.

\begin{tabular}{|c|c|c|c|c|c|c|}
\hline Autor & Año & Contaminante & $\begin{array}{c}\text { Proceso } \\
\text { Catalítico } \\
\end{array}$ & $\begin{array}{c}\text { Irradianci } \\
\left(\mathrm{W} / \mathrm{m}^{2}\right)\end{array}$ & $\begin{array}{c}\text { Tiempo } \\
\text { (min) }\end{array}$ & $\begin{array}{c}\text { Irradiació } \\
\left(\mathrm{kJ} / \mathrm{m}^{2}\right)\end{array}$ \\
\hline $\begin{array}{c}\text { Alfano } \\
y \text { col. }\end{array}$ & 2000 & $\begin{array}{c}\text { BTEX } \\
\text { (Benzene, Toluene, } \\
\text { Ethylbenzene y } \\
\text { Xylene) }\end{array}$ & $\mathrm{TiO}_{2}$ & 28 & 180 & I \\
\hline $\begin{array}{c}\text { Malato } \\
y \text { col. }\end{array}$ & $2000 \mathrm{~b}$ & Oxamyl & $\mathrm{TiO}_{2}$ & $\begin{array}{l}12,5 \\
23,4 \\
\end{array}$ & $\begin{array}{c}210(\mathrm{pH}=3) \\
204(\mathrm{pH}=7.5)\end{array}$ & $\begin{array}{l}157 \\
286 \\
\end{array}$ \\
\hline
\end{tabular}

Como puede observarse de la evolución anual de la irradiación en el OAI durante 2010 (figura 4.2), los dos contaminantes descritos por la bibliografía (BTEX y oxamyl) pueden ser degradados en cualquier mes del año. La porción de radiación no medida por el espectrofotómetro Brewer se compensa, en parte, por el mayor tiempo de iluminación y las condiciones de cielo despejado. Para visualizar mejor este hecho, los datos de irradiación obtenidos de la literatura se han representado en la figura 4.2 junto a los valores experimentales medidos en el OAI. Estos valores medios mensuales oscilan entre un valor mínimo de $404 \pm 16 \mathrm{~kJ} / \mathrm{m}^{2}$ en el mes de diciembre y un valor máximo de $966 \pm 38 \mathrm{~kJ} / \mathrm{m}^{2}$ en el mes de junio, siendo la media anual $687 \pm 211 \mathrm{~kJ} / \mathrm{m}^{2}$.

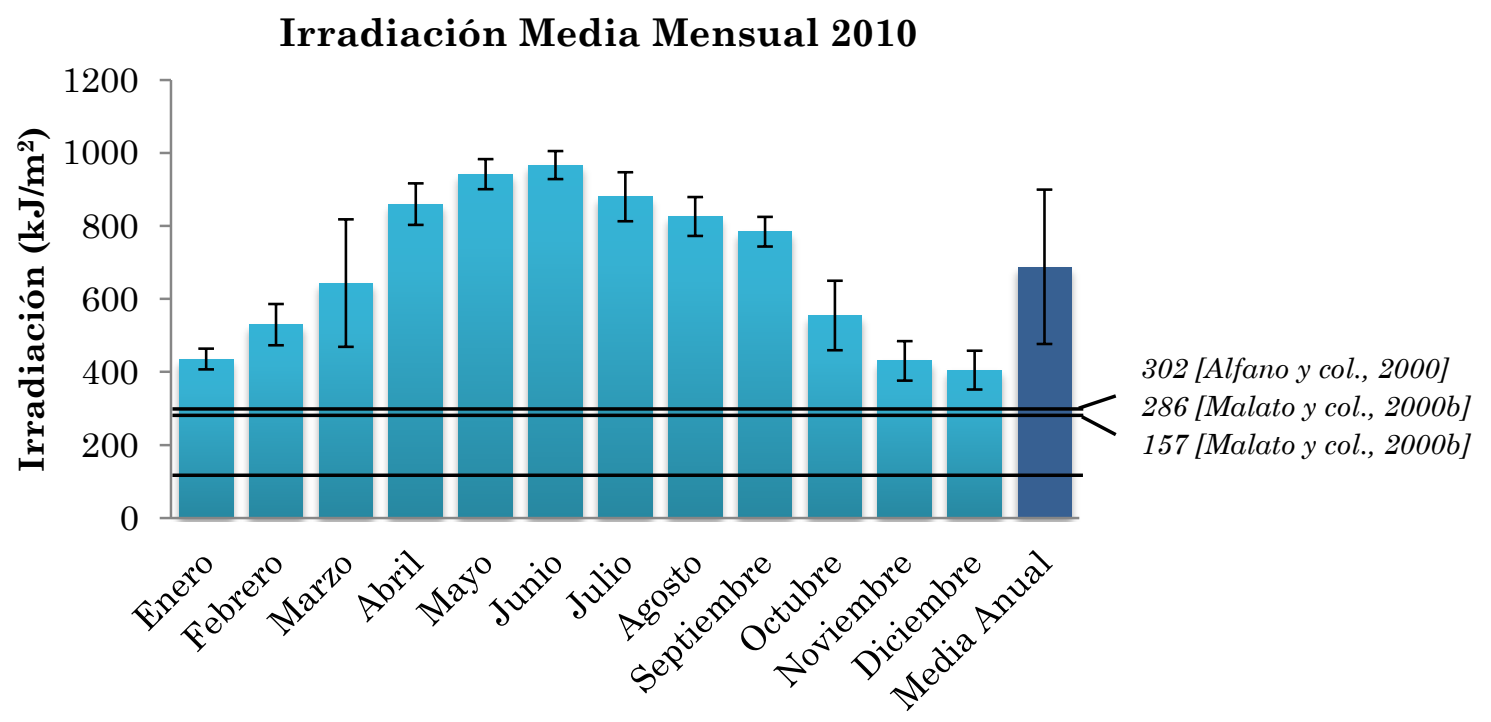

Figura 4.2 : Valores medios mensuales de irradiación global UV $\left(\mathrm{kJ} / \mathrm{m}^{2}\right)$ en el OAI para el año 2010 y valores de irradiación encontrados en la literatura. Las barras de error indican $\pm 1 \sigma(\sigma$, desviación estándar). 


\subsection{Energía acumulada por unidad de volumen, Quv}

Dentro de los parámetros más empleados en la bibliografía se encuentra el concepto de Quv,n (energía acumulada por unidad de volumen, ecuación 4.3, en kJ/l). La International Union of Pure and Applied Chemistry (IUPAC) recomienda comparar sistemas solares basándose en el área del colector (A) necesaria para conseguir un objetivo determinado en la unidad de tiempo, con lo que se llega a la siguiente definición del Quv,n:

$$
Q_{U V, n}=Q_{U V, n-1}+\Delta t n \cdot U_{V G, n}\left(\frac{A_{C P C}}{V_{T O T}}\right)
$$

(Ecuación 4.3)

donde

$\begin{array}{lll}t_{n} & \rightarrow & \text { Tiempo experimental para cada muestra } \\ U V_{G, n} & \rightarrow & \text { a radiación } U V_{G} \text { media durante } \Delta t_{n} \\ A_{C P C} & \rightarrow & \text { La superficie del colector } \\ V_{T O T} & \rightarrow & \text { Volumen total de la planta } \\ Q_{U V, n} & \rightarrow & \text { Energía acumulada por unidad de volumen }(\mathrm{kJ} / \mathrm{l}) \text { incidente en el reactor } \\ & & \text { para cada muestra durante el experimento. }\end{array}$

Para el Quv,n la tabla 4.2 resume los valores encontrados en la literatura.

Tabla 4.2: Estudios para la energía acumulada por unidad de volumen, Quv.

\begin{tabular}{ccccccc}
\hline Autor & Año & Contaminante & $\begin{array}{c}\text { Proceso } \\
\text { Catalítico }\end{array}$ & Quv (kJ/l) \\
\hline Malato $y$ col. & $2000 \mathrm{a}$ & $\begin{array}{c}\text { Acrinathrin } \\
\text { (Pesticida comercial) }\end{array}$ & $\mathrm{TiO}_{2}$ & $\mathbf{7 8 , 5}$ & $\mathbf{2 1 , 6 ( \mathrm { pH } = 7 . 5 )}$ \\
\hline Malato $y \mathrm{col}$ & $2000 \mathrm{~b}$ & Oxamyl & $\mathrm{TiO}_{2}$ & $\mathbf{1 2 , 6 ( \mathrm { pH } = 3 )}$ \\
\hline Malato $y \mathrm{col}$ & $2000 \mathrm{c}$ & $\begin{array}{c}\text { Pesticidas } \\
\text { comerciales }\end{array}$ & $\mathrm{TiO}_{2}$ & $\mathbf{5 6 , 4}$ \\
\hline
\end{tabular}

Dado que el Quv,n depende del área del colector y del volumen total de la muestra, la comparación con la literatura debe hacerse en base a los datos de irradiación mensuales (figura 4.2). Si comparamos estos datos con la bibliografía (tabla 4.3) se observa que es posible degradar todos los contaminantes descritos, dependiendo de las cantidades que se tengan de los mismos. Así, por ejemplo, con la irradiación media anual $\left(687 \pm 211 \mathrm{~kJ} / \mathrm{m}^{2}\right)$, se podrían degradar $8,8 \mathrm{l} / \mathrm{m}^{2}$ de agua contaminada con acrinathrin $(78,5 \mathrm{~kJ} / \mathrm{l}), 31,8$ o $54,5 \mathrm{l} / \mathrm{m}^{2}$ de agua contaminada con oxamyl (dependiendo del $\mathrm{pH}$ ) y $12 \mathrm{l} / \mathrm{m}^{2}$ de agua contaminada con otros pesticidas comerciales $(56,4 \mathrm{~kJ} / \mathrm{l})$. 


\subsection{Tiempo de iluminación, t30w}

En algunos casos es necesario mostrar los resultados en términos de tiempo de iluminación en lugar de en términos de irradiación o energía acumulada, Quv. No obstante, el uso de este tiempo como unidad de cálculo puede originar errores en la interpretación de los resultados, puesto que un reactor está compuesto de elementos iluminados y no iluminados. Por ello, el tiempo de iluminación normalmente se proporciona respecto a un valor de referencia de $30 \mathrm{~W} / \mathrm{m}^{2}$, que es aproximadamente la radiación solar ultravioleta media en un día soleado durante dos horas alrededor del mediodía [Radjenovic y col., 2010; Jimenez y col., 2010; Klamerth y col., 2010; Malato y col., 2000b, 2002 y 2003; Zapata y col., 2010]. Bajo esta condición el tiempo de iluminación calculado se denomina t30w.

$$
t_{30 w}=\frac{1}{I_{G U V}^{0}} \int_{\text {tinicial }}^{\text {tfinal }} I_{G U V}(t)
$$

donde

(Ecuación 4.4)

$$
\begin{array}{lll}
I_{G U V}^{o} & \rightarrow & \text { Irradiacia global UV estándar }\left(=30 \mathrm{~W} / \mathrm{m}^{2}\right) \\
I_{G U V}(t) & \rightarrow & \text { Irradiancia global UV incidente en un tiempo } t
\end{array}
$$

Aplicando la ecuación 4.4 se obtuvieron las medias mensuales de t30w mostradas en la figura 4.3, donde puede observarse que los valores mensuales oscilan entre un valor mínimo de $225 \pm 9$ min en el mes de diciembre y un valor máximo de $537 \pm 21$ min en el mes de junio, siendo la media anual $382 \pm 117 \mathrm{~min}$.

Media Mensual t t30w $_{2010}$

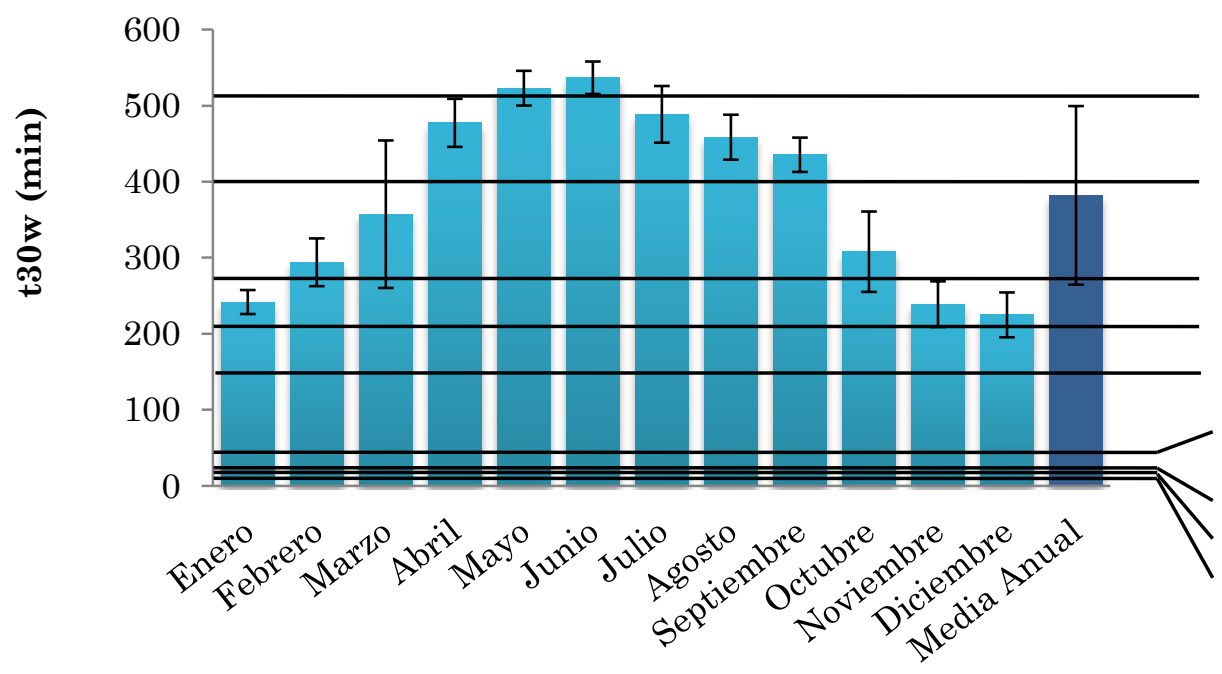

510 [Malato y col., 2000a]

400 [Zapata y col., 2010]

276 [Klamerth y col., 2010]

210 [Sirtori y col., 2011]

150 [Malato y col., 2002]

45 [Jimenez y col., 2010;

Malato y col., 2003]

22 [Radjenovic y col., 2010]

17 [Radjenovic y col., 2010]

8 [Jimenez y col., 2010]

Figura 4.3 : Valores medios mensuales de $t_{30 \mathrm{w}}$ en el OAI para el año 2010 y valores de $t_{30 \mathrm{w}}$ encontrados en la literatura. Las barras de error indican $\pm 1 \sigma$ ( $\sigma$, desviación estándar). 
$\mathrm{Al}$ igual que en los casos anteriores, los t30w encontrados en la literatura (tabla 4.3) se han representado en la figura 4.3 junto a los valores medios mensuales experimentales de $\mathrm{t}_{30 \mathrm{w}}$. Se observa que existe la posibilidad de degradar la inmensa mayoría de los contaminantes descritos a lo largo de todo el año. Las excepciones son las descritas por Malato y col. [2000a], parte de la descrita por Zapata y col. [2010] y algunos de los contaminantes emergentes descritos por Klamerth y col. [2010]. Por ejemplo, para la primera sólo llegaría a producirse la destrucción completa del contaminante en mayo y junio, ya que son necesarios 510 min para la destrucción del acrinathrin (veáse tabla 4.3). No obstante, sí se podría llegar a una degradación parcial de aproximadamente el 50\% del contaminante durante el resto del año, para lo que son necesarios tan solo $162 \mathrm{~min}$ [Malato y col., 2000a]. Por otro lado, la degradación descrita por Zapata y col. [2010], sólo llegaría a producirse en los meses más cálidos, ya que son necesarios de 40 a 400 min para la destrucción de los pesticidas comerciales dependiendo de las condiciones de reacción.

Tabla 4.3 : Estudios para $\mathrm{t}_{30 \mathrm{w}}$.

\begin{tabular}{|c|c|c|c|c|}
\hline Autor & Año & Tipo de Contaminante & $\begin{array}{c}\text { Proceso } \\
\text { Catalítico }\end{array}$ & $\begin{array}{c}\mathrm{t}_{30 \mathrm{~W}} \\
(\mathrm{~min})\end{array}$ \\
\hline Malato y col. & $2000 \mathrm{a}$ & $\begin{array}{c}\text { Acrinathrin } \\
\text { (Pesticida comercial) }\end{array}$ & $\mathrm{TiO}_{2}$ & 510 \\
\hline Malato y col. & 2002 & Pesticidas solubles en agua & $\begin{array}{c}\mathrm{TiO}_{2} \\
\text { Fenton }\end{array}$ & $\begin{array}{l}<150 \\
<150\end{array}$ \\
\hline Malato y col. & 2003 & $\begin{array}{c}\text { Diuron } \\
\text { (Herbicida) }\end{array}$ & $\begin{array}{c}\mathrm{TiO}_{2} \\
\text { Fenton }\end{array}$ & $\begin{array}{l}45 \\
45\end{array}$ \\
\hline Jimenez y col. & 2010 & $\begin{array}{c}\text { Hierbamina Gesaprim } \\
\text { (Herbicidas) }\end{array}$ & Fenton & $\begin{array}{c}45 \\
8\end{array}$ \\
\hline Klamerth y col. & 2010 & Contaminantes Emergentes & Fenton & 43 a 276 \\
\hline Radjenovic y col. & 2010 & $\begin{array}{c}\text { Raditinide } \\
\text { (Distilled Water) }\end{array}$ & $\begin{array}{c}\mathrm{TiO}_{2} \\
\text { Fenton }\end{array}$ & $\begin{array}{c}22 \\
17,4\end{array}$ \\
\hline Zapata y col. & 2010 & Pesticidas comerciales & Fenton & 40 a 400 \\
\hline Sirtori y col. & 2011 & Ácido Nalidixic & Fenton & 210 \\
\hline
\end{tabular}




\section{Conclusiones}

En vista de los resultados anteriores se puede concluir que la fotocatálisis de contaminantes es posible, para la inmensa mayoría de los casos descritos en la literatura, con la radiación solar ultravioleta registrada en el Observatorio Atmosférico de Izaña. Esto es debido a que la ubicación del OAI favorece que se registren valores muy altos de esta radiación, asociados a los valores relativamente bajos de ozono total en columna, a la elevada altitud de la estación, la poca presencia de nubes y aerosoles atmosféricos. Sin embargo, las condiciones experimentales encontradas en la literatura son muy variadas, por lo que no es posible realizar una comparación homogénea en todos los casos.

Este trabajo es un estudio preliminar para evaluar la posibilidad del tratamiento de aguas mediante TOAs Fotocatalíticos en el OAI, pero puede servir de punto de referencia o partida para otros lugares de la isla de Tenerife. No obstante, cabe destacar que la situación de la isla de Tenerife en el Océano Atlántico, en el eje de la franja subtropical y al oeste del continente africano, así como su orografía le confiere unos rasgos climáticos específicos. La altitud y la orientación del relieve inducen diferencias en la distribución espacial de los elementos climáticos, principalmente de la precipitación, la temperatura y la nubosidad, haciendo que sea posible registrar datos de radiación muy diferentes de una zona a otra. Por ello, es importante que en futuros análisis el equipo de medida se encuentre en el emplazamiento del sistema de depuración. Aunque, en el caso de no disponer de medidas experimentales, la radiación solar puede ser estimada a partir de modelos numéricos que simulan la transferencia radiativa en la atmósfera.

Finalmente, es muy interesante destacar que la técnica de medida del espectrofotómetro Brewer podría ser de mucha utilidad en la investigación de nuevos fotocatalizadores aplicables a los TOAs. Este equipo proporciona medidas espectrales de alta calidad de la radiación solar, las cuales pueden ser comparadas con los espectros de absorción de dichos fotocatalizadores, pudiéndose estudiar el grado de descomposición de los contaminantes por efecto de la luz solar de forma simultánea. 


\section{Agradecimientos}

Este trabajo ha sido realizado en el contexto de la beca de formación "Centro Europeo de Calibración Brewer: formación en técnicas de medida estándares y nuevas técnicas de medida con espectrofotómetros Brewer" de la Agencia Estatal de Meteorología (AEMET). Asimismo, nos gustaría agradecer a los observadores meteorológicos del OAI sus chequeos rutinarios a los espectrofotómetros Brewer y al personal de mantenimiento, su colaboración. 


\section{Referencias}

Acosta. L., "Proceso Fotofenton de Oxidación Quimica para el Tratamiento de aguas Industriales", Tesis doctoral año 2009, Departamento de Ciencia e Ingeniería de Materiales e Ingeniería Química. Universidad Carlos III de Madrid.

Alfano, O.M.; Bahnemann, D.; Cassano, A.E.; Dillert, R. ; Goslich, R., "Photocatalysis in water environments using artificial and solar light”, Catalysis Today 58 (2000) 199-230.

Andreozzi, R.; Caprio, V.; Insola, A.; Marotta,R., “Advanced oxidation processes (AOP) for water purification and recovery”, Catalysis Today 53 (1999) 51-59.

Bahnemann, D., “Photocatalytic water treatment: solar energy applications”, Solar Energy 77 (2004) 445-459.

Bais, A.F.; Kazadzis, S.; Balis, D.; Zerefos, C. S.; Blumthaler, M., “Correcting global solar ultraviolet spectra recorded by a Brewer spectroradiometer for its angular response error", Applied Optics 37 (1998).

Belgiorno, V.; Rizzo, L.; Fatta, D.; Della Rocca, C.; Lofrano, G.; Nikolaou, A.; Naddeo, V.; Meri,S., "Review on endocrine disrupting-emerging compounds in urban wastewater: occurrence and removal by photocatalysis and ultrasonic irradiation for wastewater reuse", Desalination 215 (2007) 166-176.

Blanco, J.; Malato, S.; Fernández-Ibañez, P.; Alarcón, D.; Gernjak, W.; Maldonado, M.I., “Review of feasible solar energy applications to water processes”, Renewable and Sustainable Energy Reviews 13 (2009) 1437-1445.

Borges, M. E.; Alvarez-Galván, M.C.; Esparza, P.; Medina, E.; Martín-Zarza, P.; Fierro, J.L.G., “Ticontaining volcanic ash as photocatalyst for degradation of phenol”, Energy and Environmental Science 1 (2008) 364-369.

EPA, “US/EPA Handbook of Advanced Photochemical Oxidation Processes”, EPA/625/R98/004, 1998.

Esparza, P.; Borges, M. E. ; Díaz, L., "Studies in a Fixed-Bed Photocatalytic Reactor System Using Natural Materials for Degradation of a Dye Contaminant in Water”, Water Air Soil 
Esparza, P.; Borges, M.E.; Díaz, L. ; Alvarez-Galván, M.C. ; Fierro, J.L.G., "Photodegradation of dye pollutants using new nanostructured titania supported on volcanic ashes", Applied Catalysis A: General 388 (2010) 7-14.

Farias, J.; Albizzati, E.D.; Alfano, O.M., "New Pilot-Plant Photo-Fenton Solar Reactor for Water Decontamination”, Industrial and Engineering Chemistry Research 49 (2010) 1265-1273.

Fioletov, V.E; MCArthur, L; Kerr, J.B; Wardle, D.I, " A long-term variations of UV-B irradiance over Canada estimated from Brewer observations and derived from ozone and pyrometer measurements”, Journal of Geophysical Research 106 (2001) 23009-23028.

GrÖbner, J.; Melet, C., "Aerosol optical depth in the UVB and visible wavelength range from Brewer spectrophotometer direct irradiance measurements: 1991-2002", Journal of Geophysical Research 109 (2004).

Hansen, K.K., “A study of 1998-2008 Brewer Mk V Spectrophotometer UV-measurements in Oslo", Tesis doctoral año 2009, Departamento de Física. Universidad de Oslo.

Herrmann, J.M., "Heterogeneous photocatalysis: fundamentals and applications to the removal of various types of aqueous pollutants", Catalysis Today 53 (1999) 115-129.

Jiménez, M; Oller, I.; Maldonado, M.I.; Malato, S.; Hernández-Ramírez, A.; Zapata, A.; PeraltaHernández, J.M., "Solar photo-Fenton degradation of herbicides partially dissolved in water”, Catalysis Today 161 (2010) 214-220.

Kerr, J.B; MCElroy, C.T; Olafson, R.A, "Measurements of ozone with the Brewer spectrophotometer, Proceedings of the Quadrennial International Ozone Symposium", National Center for Atmospheric Research (1981) 74-79

Klamerth, N.; Rizzo, L.; Malato, S.; Maldonado, M.I.; Agüera, A.; Fernández-Alba, A.R., "Degradation of fifteen emerging contaminants at mg LL1 initial concentrations by mild solar photo-Fenton in MWTP effluents”, Water research 44 (2010) 545 - 554.

Malato, S.; Blanco, J.; Fernández-Alba, A.R.; Aguera, A., “Solar photocatalytic mineralization of commercial pesticides: Acrinathrin”, Chemosphere 40 (2000a) 403-409.

Malato, S.; Blanco, J.; Richter, C.; Fernández, P.; Maldonado, M.I., "Solar photocatalytic mineralization of comercial pesticides: Oxamyl”, Solar Energy Materials and Solar Cells 64 (2000b) 1-14.

Malato, S.; Blanco, J.; Richter, C.; Maldonado, M.I., "Optimization of pre-industrial solar photocatalytic mineralization of commercial pesticides Application to pesticide container recycling", Applied Catalysis B: Environmental 25 (2000c) 31-38.

Malato, S.; Blanco, J.; Cáceres, J.; Fernández-Alba, A.R.; Agüera, A.; Rodríguez, A., "Photocatalytic treatment of water-soluble pesticides by photo-Fenton and TiO2 using solar energy", Catalysis Today 76 (2002) 209-220.

Malato,S.; Cáceres,J.; Fernández - Alba, A.R.; Piedra, L.; Hernando, M.D.; Aguera, A.; Vial, J., "Photocatalytic Treatment of Diuron by Solar Photocatalysis: Evaluation of Main Intermediates and Toxicity”, Environmental Science and Technology 37 (2003) 2516-2524. 
Malato, S.; Fernández-Ibáñez, P. ; Maldonado, M.I. ; Blanco, J. ; Gernjak, W., “Decontamination and disinfection of water by solar photocatalysis: Recent overview and trends”, Catalysis Today 147 (2009) 1-59.

Michael, I.; Hapeshi, E.; Michael,C.; Fatta-Kassinos, D., "Solar Fenton and solar TiO2 catalytic treatment of ofloxacin in secondary treated effluents: Evaluation of operational and kinetic parameters", Water research 44 (2010) 5450-5462.

Mills, A.; Le Hunte, S., “An overview of semiconductor photocatalysis”, Journal of Photochemistry and Photobiology A: Chemistry 108 (1997) 1-35.

Nogueira, R; Jardim, W.F., “TiO2-fixed-bed reactor for water decontamination using solar light”, Solar Energy 56 (1996) 471-477.

Oyama, T.; Aoshima, A.; Horikoshi, S.; Hidaka, H.; Zhao, J.; Serpone, N. "Solar photocatalysis, photodegradation of a commercial detergent in aqueous TiO2 dispersions under sunlight irradiation”, Solar Energy 77 (2004) 525-532.

Pozzo, R.L.; Baltanfis, M.A.; Cassano, A.E., "Supported titanium oxide as photocatalyst in water decontamination: State of the art”, Catalysis Today 39 (1997) 219-231.

Radjenovic, J.; Sirtori, C.; Petrovic, M.; Barceló, D.; Malato,S., “Characterization of intermediate products of solar photocatalytic degradation of ranitidine at pilot-scale”, Chemosphere 79 (2010) 368-376.

Redondas, A., “Curso de Operadores de Espectrofotómetros Brewer”, Comunicación interna (2000).

SCI-TEC Instruments Inc., “MKIII Operator's Manual”, (1998).

Shawaqfeh, A. T.; Al Momani, F. A., "Photocatalytic treatment of water soluble pesticide by advanced oxidation technologies using UV light and solar energy", Solar Energy 84 (2010) 1157-1165.

Sirtori, C.; Zapata, A.; Gernjak, W.; Malato, S.; Lopez, A.; Agüera,A., "Solar photo-Fenton degradation of nalidixic acid in waters and wastewaters of different composition. Analytical assessment by LCeTOF-MS", Water Research 45 (2011) 1736-1744.

Webb, A. R., “UV instrumentation for field and forest research", Agricultural and Forest Meteorology 120 (2003) 27-38.

Zapata, A.; Oller I. ; Rizzo, L. ; Hilgert, S. ; Maldonado, M.I. ; Sánchez-Pérez, J.A. ; Malato S., "Evaluation of operating parameters involved in solar photo-Fenton treatment of wastewater: Interdependence of initial pollutant concentration, temperature and iron concentration”, Applied Catalysis B: Environmental 97 (2010) 292-298.

Zerefos, C.; Bais, A.F.; Zerefos, C.F., "Solar Ultraviolet Radiacion: Modelling, Measurements and Effects", Springer (1997). 